\title{
Téoros
}

Revue de recherche en tourisme

\section{Le parc marin du Saguenay-Saint-Laurent : écotourisme marin}

\section{Bernard Maltais}

Volume 14, numéro 1, printemps 1995

Les parcs : des réseaux en mutation

URI : https://id.erudit.org/iderudit/1077059ar

DOI : https://doi.org/10.7202/1077059ar

Aller au sommaire du numéro

Éditeur(s)

Université du Québec à Montréal

ISSN

0712-8657 (imprimé)

1923-2705 (numérique)

Découvrir la revue

Citer cet article

Maltais, B. (1995). Le parc marin du Saguenay-Saint-Laurent : écotourisme marin. Téoros, 14(1), 52-54. https://doi.org/10.7202/1077059ar d'utilisation que vous pouvez consulter en ligne.

https://apropos.erudit.org/fr/usagers/politique-dutilisation/ 


\title{
Le parc marin du Saguenay-Saint-Laurent: écotourisme marin
}

\author{
Bernard Maltais"
}

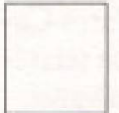

La mer, source inépuisable de ressources; dernière des utopies humaines démasquées! Car ce n'est pas un hasard si la création des pares nationauxcanadiens cö̈ncideavec la prise de conscience des gouvernements del'importance de la conserwation des ressources marines. Les pêcheries maritimes, dotéesd'une technologie sophistiquée, ont prélevé un lourd tribut sur les richesses de la mer. Déjà, la rareté de certaines espèces de baleines ou la disparition d'autres espèces annonçait que la mécanique humaine aurait raison de la mer et de ses ressources. Les Basques, avec leurs maigres moyens, puisqu'ils devaient pourchasser au $X V^{t}$ et XVIt siècles la baleine noire en canot à rames, avaient éradiqué cette espèce du golfe du Saint-Laurent. Plus récemment, ce fut le béluga qui eut à subir une chasse qui préleva un lourd tribut sur le troupeau du Saint-Laurent. La destruction de son habitat et la contamination de son milieu de vie ont par ailleurs affaibli le troupeau. Affligé de tous les maux de la pollution et malgré les mesures de protection dont il fait Pobjet, les recherches actuelles ne permettent pas de savoir avec certitude si le troupeau de bélugas du Saint-Laurent diminue, se maintient ou augmente.

Pourquoi aura-t-il fallu attendre si longtemps pour préserver une ressource qui a façonné des cultures et présidé à l'émergence de civilisations? Pourtant, I'hommeavait fait preuvedesagesse devant la dégradation des paysages grandioses de l'Ouest américain en préservant de l'exploitation humaine des espaces inaltérés grâce à la création de parcs nationaux. La mer se prête mal à l'investigation humaine. Cet univers à trois dimensions, où les lois terrestres ne s'appliquent pas, est longtemps demeuré une énigme. Ce n'est en effet que depuis un peu plus de 50 ans que nous pouvons accéder physiquement à ses fonds, grâce à l'invention du scaphandre autonome, etconstater de visu ses richesses. Cette nouvelle conquête des lieux marins a permis à l'homme de réaliser qu'ils souffraient des mêmes maux que les paysages

Monsieur Bernard Maltais est directeur de la Direction de la planification stratégique et des polltiques (DPSP) a Patrimoine Canadien - Fiegion du Québec. terrestres; dégradation des fonds marins et du milieu de vie, surexploitation des ressources vivantes... Ce n'est que devant ces constats que la communauté internationale incita les états côtiers à prendre toutes les mesures nécessaires pour préserver les précieuses ressources de la mer.

Faute de mieux, ces états commencèrent à créer des parcs nationaux marins, dont le concept était calquésur les pares nationaux terrestres. Ceconceptdes espacesprotégés demeure toutefoisinadaptéá la préservation des ressources en milieu marin. En effet, les vastes écosystèmes et leur perméabilité relative créent des conditions de vie fort différentesde celles existant sur terre. Ainsi, plusieurs espèces comptent sur les courants marins pour la dissémination de leurs oeufs et les mammiferes marins mettent bas dans les eaux chaudes des tropiques et trouvent dans les mers plus nordiques des aires d'alimentation et d'élevage appropriées. Leur migration annuelle peut s'effectuerà l'échelle des hémisphères de la planète. Certainesespècestraversentmême la barrière des eaux douces et des eaux salées, comme le saumon anadrome et languille catadrome. Du point de vue de leur conservation, une aire d'alimentation ou de reproduction saine n'est pas garante de leur maintien. Car ce qu'il faut retenir c'estqu'uneaire contaminée, quellequ'elle soit, aura_un effet néfaste sur le cycle de vie des espèces. Tout espace préservé ne peut seul prétendre assurer le maintien de la vie des espèces dont l'aire vitales'étend parfois sur des millions de kilomètres carrés. En outre, la contamination, qu'elle soit terrestre ou marine, aura tốt ou tard un effet nuisible à l'aval. La pollution recouvre ainsi comme d'un linceul ce qui demeure, aujourd'hui encore, la source de la vie sur terre.

Seule solution efficace et définitive à la contamination des eaux, le tarissement des sources de pollution est une autre utopie de cette fin de siècle, car elle présente un coût socioéconomique trop important pour être envisagé. Ainsi, le concept de développement durable s'avère-t-il incontournable car, bien que l'on reconnaisse la nécessité du maintien de la diversité biologique, il faut y arrimer le bien-être des sociétés humaines qui, lui, engendre les activités et ledéveloppementéconomiquegénérateurs de répercussions environnementales considérées nuisibles.

La création de parcs marins ne soustrait donc pas, comme c'est le cas des parcs nationaux terrestres, les ressources qu'ils recèlent des répercussions de l'activité humaine. Mais elleattirel'attention du public sur la fragilitédes ressourceset l'impérieuse nécessité de les mieux gérer et d'apporter une contribution à leur conservation, au bénéfice des générations actuelles et futures. $\mathrm{La}$ mise en réserve d'aires marines demeure une bien faible contribution à la préservation des ressources, et c'est d'ailleurs qu'il faut chercher leur véritable raison d'être.

Les parcs marins possèdent le pouvoir de l'éwocation, de la démonstration. Ils ont surtout le pouvoir d'attirer l'attention sur des phénomènes et des processus physiques ou chimiques et de réinventer un savoir populaire forgé sous l'empire de l'imaginaire. C'est dans cette perspective qu'il faut envisager le tourisme dans les parcs marins et le parc marin du SaguenaySaint-Laurent n'echappe pas à cette règle.

\section{Tourisme traditionnel au parc marin du Saguenay-Saint-Laurent}

La région du pare marin du SaguenaySaint-Laurent hérite d'un engouement touristique enviable par rapport aux autres régions du Québec. En effet, Tadoussac, situé au confluent du fjord du Saguenay et du Saint-Laurent, était, avec La Malbaie, une destination touristique courue par la sociétéanglo-saxonne fortunéede l'Ontario et de la Nouvelle-Angleterre depuis le tournant du siècle jusque vers 1960 . A l'abandon des croisières proposées par le Canada Steamship Line, ont survécu les résidences secondaires et une fréquentation estivale de riches visiteurs encore fascinés par la beauté des lieux. Station balnéaire classique où, loin de l'agitation urbaine, vivait une petite communauté profitant d'un repos saisonnier bien mérité, Tadoussac devint, grâce à la démocratisation des loisirs, une étape sur le circuit touristique en développement de la Côte- 
Nord en raison de la présence d'une salmoniculture et d'un hôtel de renom.

D'étape touristique, Tadoussac est redevenue une destination lorsqu'un nouveau produit touristique vit le jour au début des années 1980, alors que des promoteurs proposèrent à leur clientèle de partir à la découverte des mammiferes marins de l'estuaire. Malgré des débuts modestes, c'est maintenant une véritable industrie touristique offrant une gamme complète de services quis'estinstalléeà Tadoussacet $c e$, en moins de 10 ans. Cetengouement du public pour les croisières d'observation aux baleines n'est pas étranger aux préoccupations écologiques et aux campagnes des groupes environnementaux favorables à la protection des troupeaux de mammiferes marins. Les caractéristiques océanographiques, la présence constante ainsi que la concentration d'espèces considérées rares ou spectaculaires dans la région de l'embouchure du Saguenay ont contribué à affermir cette région comme destination baleine.

A cet égard, il faut noter que le secteur accueille régulièrement près de onze espèces dont des pbocidaes (phoques communs, gris et du Groënland), des dauphins et marsouins et des rorquals. De ces espèces, quatre sont principalement recherchées par les touristes et pour cause. D'abord, les deux espèces qui sont l'objet des croisiéristes: le petit rorqual, généralement solitaire, et le rorqual commun qui forment des groupes compacts pouvant compter jusqu'à une douzaine d'individus. Cesdeuxespéces fréquententgénéralement les mers tempérées. Puis, deux espèces polaires beaucoup plus spectaculaires soit le rorqual bleu, espèce de haute mer et le plus grand animal ayant existé sur la planète, etle béluga, petite baleine blancherésidente de l'estuaire, dont la précarité interdit aux croisiéristes toute recherche active. La présence simultanée de ces quatre espèces, constante en saison estivale et au début de l'automne, est unique au monde et constitue le produit touristique de la région de l'embouchure du Saguenay.

Avec un tel produit touristique, les promoteurs ne tardèrent pas à solliciter la clientèle internationale. Les efforts de promotion se firent d'abord sur le marché français, marché naturel pour les promoteurs québécois. Ce furent les aubergistes de Tadoussac et de Charlevoix, puis les Associations touristiques régionales (ATR) et les opérateurs qui participèrent à des foires commerciales, oomme celle d'Atelier Canada à Paris. Sur ce plancher se transigent forfaits canadiens aux baleines ou séjours à la cabane à sucre... La participation des promoteurs de Tadoussac proposant un produit touristique moteur faisant appel, entre autres, à l'imaginaire populaire, et l'association du parc marin du SaguenaySaint-Laurent aux efforts des promoteurs, ont permis de détourner une partie de la clientèle française férue de grands espaces et des stéréotypes canadiens que sont l'Ouest, la faune sauvage, les montagnes rocheuses... Les promoteurs du Saguenay n'ont, jusqu'à présent, fréquenté que le marché français. Leurs efforts promotionnels ont, bien entendu, permis d'augmenter le contingent d'étrangers dans le parc en plus d'allonger la saison de fréquentation de la région à septembre et octobre.

Le forfait baleine attire une large clientèle et nul doute qu'une campagne de promotion sur des marchés européens moins traditionnels que le marché français augmenterait encore les cohortes étrangères. Déjà Italiens ou Allemands, ayant acquis des forfaits baleines des grossistes français présents à Atelier Canada ou en visite au pays, débarquent aujourd'hui à Tadoussac. Reste la clientèle américine, notamment celle de la Nouvelle-Angleterre située à proximité par rapport aux clientèles européennes, qui pourrait constituer un bassin fort intéressant. Mais voilà, les Américains du nord-estdes Etars-Unis constituent une clientèle quasi captive de ce genre de produit touristique car la région de Cape Cod accueille en début d'été petits rorquals, rorquals communs et rorquals à bosses, dont les bouffonneries sont pour le moins spectaculaires.

\section{Tourisme grandeur nature}

Parallèlement à la croissance des marchés et des fréquentations, le produit proposé aux visiteurs a évolué. «Baleines à 3 heuress annonçait le capitaine du navire, et c'est en rafale que les cliquetis des appareils photographiques coupaient net toutes conversations à bord. On se ruait littéralement à tribord ou à babord. Tous scrutaient la mer et l'horizon dans l'espoir d'être les-premiers à indiquer aux autres la présence d'une ou de plusieurs baleines. Le flair des observateurs tenait lieu de connaissances. L'excursionniste revenait heureux d'avoir vu, et d'avoir immortalisé une rencontre furtive avec les colosses de la mer et d'être devenu, l'espace d'un instant, celle ou celui qui a su repérer un petit rorqual ignoré de tous, ou remarqué la gigantesque baleine bleue à l'horizon.
À l'observation pure est venue se greffer une série d'informations sur la biologie des espèces, leur distribution et leur comportement. Des naturalistes à bord des navires d'observation diffusent dorénavant leurs connaissances et expliquent aux excursionnistes les raisons d'une telle concentration et la diversité des mammiferes marins dans la région de l'embouchure du Saguenay. Ces naturalistes, embauchés par les propriétaires des bateauxd'excursion, mettent ainsi à profit la relation étroite entre la configuration des fonds marins, la dynamique des courants, l'abondance du krill (euphosidae) etd'autres espèces. Les rorquals en migration viennent chaque année profiter de cette abondante source d'alimentation. Pour le béluga du Saint-Laurent, l'excursionniste apprend que cette espèce, contrairement aux autres, est résidente du fleuve et témoigne d'un passé glaciaire récent à l'échellegéologique. C'està partir de ces concepts de base que sont construits des scénarios d'interprétation de la nature et de ses ressources mettant en valeur le comportement des espèces, leur cycle de vie, etc. Les recherches scientifiques sont également utilisées comme vecteur d'interprétation et il n'est pas rare qu'un naturaliste diffuse et valorise les hypothèses des recherches en cours et qu'il en livre les résultats aux excursionnistes.

\section{Tourisme écologique}

La croisière aux baleines à bord de navire de tout gabarit est toutefois victime de ses succès. L'engouement du public à la recherche de sensations toujours de plus en plus fortes et son désir d'accéder à la ressource de plus en plus rapidement ont occasionné l'acquisition de bateaux plus petits (les zodiacs de mer), à partir desquels le visiteur peut observer au ras de l'eau les mammiferesmarins. Par ailleurs, plusieurs propriétaires de petits bateaux furent tentés par l'aventure et s'inscrivirent à la liste défà longue des promoteurs. Bien que plusieurs soient sensibles à la délicatesse des approches, cette profusion de prestataires de services de croisières d'observation engendre un trafic maritime que certains considèrent préjudiciable à la ressource et à la sécurité du public.

Enoutre, il faut noter que, bien souvent, les observations ont lieu en plein coeur de la voie de navigation internationale du SaintLaurent où passent tankers, porte-containers et navires marchands dont les horaires sont prioritaires. Par temps gris, lerepérage des petits navires ajoute aux difficultés de navigation dans ce secteur. Afin d'assurer la protection de la ressource, de sensibiliser 
les capitaines de navires aux résultats des recherches sur les mammiferes marins et deles yassocier, et de leur rappeler diverses consignes de sécurité, Pẻches et Océans Canada, en association avec l'administration du Parc marin du Saguenay-SaintLaurent tiennent, en début de saison touristique, une réunion d'information et d'échanges où sont convoqués tous les propriétaires opérateurs de croisières d'observation aux baleines. C'est ainsi qu'à l'instigation des autorités gouvernementales, les capitaines se sont dotés d'un code d'éthique qui régit en quelque sorte leur comportement lors de croisières, tant sur le plan du respect mutuel qu'ils se doivent en mer qu'en matière d'approche de la ressource. Le code prescrit certaines mesures de protection plus pointues, notamment pour le béluga du Saint-Laurent. L'industrie touristique s'engageait ainsi résolument dans levirage écologique. Bien qu'un tel codeait permis l'ordonnancement de l'observation, le grand nombre de navires proposant à leurs passagers l'observation d'une baleine ou d'un groupe de baleines rompt pour plusieurs excursionnistes l'enchantement. L'expérience du visiteur s'en trouve considérablement amoindrie.

\section{L'éveil environnemental}

Le paremarindu Saguenay-Saint-Laurent fut le premier à proposer aux amateurs de plus en plus sensibilisés à l'approche écologiste un autre type d'expérience d'observation. L'administration du parc a rendu accessibles les sites de Pointe-Noire et de Cap-de-Bon-Désir, sites qui permettent une observation directe des mammiferes marins dans des conditions parfois supérieures à celles des navires de croisière. Le promontoire de Cap-de-Bon-Désir, haut lieu de l'observation terrestre des mammiferes marins, est devenu, en quelques annéesseulement, le rendez-vousd'un public sensibilisé aux répercussions potentielles de l'intense activité maritime d'observation. De plus, pour éviter que le public visiteur du promontoire soit exposé aux mêmes contraintes d'achalandage que celles auxquelles sont confrontés les excursionnistes, les bateaux observent la consigne de ne pas pénétrer à l'intérieur d'un rayon d'un mille marin ceinturant le Capde-Bon-Désir.

La valorisation de ce site émane de la mission du ministère du Patrimoine canadien (Parcs) qui associe à la conservation l'appréciation des ressources par le public. Ainsi, en proposant une option valable à l'observation des baleines a bord de ba- teaux, Parcs Canada s'acquitte, dans une certaine mesure, de ses obligations de fiduciaire de la ressource. Le Ministère contribue en outre à l'éveil du public à la cause environnementale grâce à la transmission, sur le site, de messages visant la protection de la nature et de ses ressources.

Outre le produit baleine, le visiteur de Cap-de-Bon-Désir a l'occasion de partir à la découverte d'un monde plus secret, à l'abri des regards indiscrets, celui de la faune et de la flore sous-marines car, fautil le rappeler, le parc marin du SaguenaySaint-Laurent n'a pas pour seul objectif la préservation des baleines. En effet, c'est ['ensemble des ressources marines que ce parc préserve et les activités d'éveil environnemental utilisent des vecteurs de transferts de connaissances vers le public comme le rôle que jouent les organismes filtreurs dans l'environnement marin ou les mécanismes qui régissent la chaîne alimentaire. Ainsi, chaque activité proposée à l'appréciation du public est-elle conçue en fonction de l'acquisition de connaissances qui permettent aux visiteurs de prendre consciencedel'équilibreet desinteractions constantes des ressources et du potentiel de rupture que peuvent présenter les activités humaines. Nulleactivitéd'appréciation ne possèdecependant le pouvoir évocateur de celle des croisières d'observation. C'est dans cette perspective que levisiteur est de plus appelé à comprendre les rapports que 'homme a entretenus avecles baleines, soit l'époque où leur valeur économique était reliée à leur transformation industrielle, la période d'indifférence du public à leur égard, la diminution de leur nombre, le dépérissement et la disparition d'espèces et les efforts de protection tardifs dont certaines espèces ont bénéficié, de même que les succès et les insuccès des campagnes internationales visant leur préservation. Sont également valorisés l'apport des recherches sur les mammiferes marins à l'avancement des sciences, leur rôle dans la chaîne alimentaire et leur contribution au diagnostic del'état del'environnement. D'une simple visite, au départ divertissant ou l'inhabituel rompt le quotidien, le visiteur est sensibilisé à la richesse et à la diversité du milieu, à la complexité de l'écosystème marin, au rôle desocéans dans la régulation thermique de la planète... Levisiteur, ainsi guidé, chemineà traversle monde fabuleux et complexe d'une nature dorénavant à sa portée. Saisir la complexité de la natureou comprendre les systèmes écologiques et en apprécier les valeurs ne font toutefois pas du visiteur un gardien du patrimoine.

\section{Veille patrimoniale}

La démarche de sensibilisation du public doit être poussée beaucoup plus loin. C'est ainsi que dans le cadre des croisières d'observations des mammiferes marins, les passagers à bord des navires se font expliquer, dès leur embarquement, les principes qui guident les promoteurs dans leur quête d'observation des mammiferes marins de même que les préceptes à respecter. Les croisiéristes peuvent donc, à partir du code d'éthique régissant l'industrie, jauger les promoteurs dans l'exploitation de cette activité. Le visiteur devient donc un gardien de l'espace protégé, C'est la veille patrimoniale.

Dans ce même parc, une bonne partie de la recherche scientifique privée a été subventionnée par des contributions volontaires devisiteursou groupesayantentenduparler de la précarité du troupeau des bélugas du Saint-Laurent. Ces contributions se sont faites par l'adoption d'un béluga au coût de 5000 S (CDN). Les organismes de recherche ont ainsi pu poursuivre leurs recherches sur la population et le comportement du troupeau du Saint-Laurent. Cette contribution volontaire constitue de fait uneactivitédegardiennage patrimonial puisque, ce faisant, le visiteur participe à la recherche scientifique dont les résultats se traduisent, bien sûr, par une connaissance plus pointue du milieu, mais surtout par l'adoption de mesures de gestion visant la conservation de l'espèce.

\section{RÉFÉRENCES}

ENVIRONNEMENT CANADA, Politique sur les parcs marins nationaux, Gouvernement du Canada, Ottawa, 1986, $18 \mathrm{p}$.

GOUVEANEMENT DU CANADA, Lol sur les pares nationaux, L.A., 1985, ch. N-14.

GROUPE D'ETUDE SUR LA CREATION DE PARCS NATIONAUX D'ENVIRONNEMENT CANADA, Rapport Theberge, Parcs 2000, per spectives pour le 21" sidcle, Université de Waterloo, Ottawa, $1985,32 \mathrm{p}$

MALTAIS, Bernard, Gestion des parcs marlne natlonaux, non publis, $1967,28 \mathrm{p}$

PATAIMOINE CANADIEN, Pare marin du Saguenay. Saint-Laurent: plan de gestion, Gouvernement du Canada Quebec, 1994.

PATAIMOINE CANADIEN, Principes directeurs et politiques de gestion. Parcs Canada, Gowvernement du Canada, Ottawa, $1994,127 \mathrm{p}$.

VIEL, Annette, L'omergence de lieux du patrimoine pour veiller l'eau et lestleuves du monde, Parcs Canada, Québec, 1993,20 p., non publié. 